MATEC Web of Conferences 38, 01004 (2016)

DOI: $10.1051 /$ matecconf/20163801004

C) Owned by the authors, published by EDP Sciences, 2016

\title{
Seismic Shear Energy Reflection By Radon-Fourier Transform
}

\author{
Umairia Malik ${ }^{1, a}$, Dennis Ling Chuan Ching $^{2}$ and Hanita Daud ${ }^{3}$ \\ ${ }^{1,2,3}$ Fundamental and Applied Sciences Department, 32610,Bandar Seri Iskandar, Universiti Tekonologi PETRONAS, Malaysia.
}

\begin{abstract}
Seismic waves split in an anisotropic medium, instead of rotating horizontal component to principal direction, Radon-Fourier is derived to observe the signature of shear wave reflection. Synthetic model with fracture is built and discretized using finite difference scheme for spatial and time domain. Common depth point (CDP) with single shot gives traces and automatic gain is preprocessed before Radon Transform (RT), a filtering technique gives radon domain. It makes easier to observe fractures at specific incidence and improves its quality in some way by removing the noise. A comparison of synthetic data and BF-data is performed on the basis of root means square error (RMS) values. The RMS error is minimum at the $10^{\text {th }}$ trace in radon domain.
\end{abstract}

\section{Introduction}

Continuous progress in geophysical exploration techniques, searching for hydrocarbon reservoirs, mud rock cracks, volcanic rocks and minerals have become new goals for exploration. Vertical seismic profiling (VSP) technique plays a pivotal role in the geophysical exploration and measures the upgoing and down going seismic wave fields through the subterranean region. So the multi-component borehole exploration is successfully used in determing the medium properties like fracture density, Poisson's ratio and anisotropy, etc. Shear wave splitting is one of the most manifestation behaviors of waves in anisotropic medium also called birefringence. Shear waves splits into two orthogonal polarizations, $S_{V}$ and $\mathrm{S}_{\mathrm{H}}$ that travel at different velocities and have singling out seismic signatures. Generally the orientations of polarizations yield information about the anisotropic symmetry and stress directions, whereas time exhibit average crack's density along the ray paths [1].

In simple isotropic medium $\mathrm{S}_{\mathrm{V}}$ is polarized in the plane of propagation while the $\mathrm{S}_{\mathrm{H}}$ is orthogonal to it. In the presence of layered medium, $S_{\mathrm{V}}$ has polarization in radial (source-receiver) direction, and $S_{H}$ would be in transverse direction [2]. Thus the azimuthally aligned shear wave splitting is observed almost in sedimentary rock [3] and is a sort of two-edged sword that also indicates the fractures in reservoirs.

Shear wave splitting (SWS) came forth prominently for characterizing anisotropy in earth after the initial work of Keith and Crampin [4]. Ando et al. [5], Vinnik et al. [6], Fukao [7], and Silver and Chan [8]. Numerous methods have been established for measuring the shear wave splitting i.e. display techniques, automatic techniques, semi automatic technique, linearity techniques and artificial neural network techniques.
Measuring SWS accurately is a challenging task because of complexity of shear wave signal thus all the techniques have some pros and cons [9].

Display techniques consist of polarisation diagrams, rotated seismograms, vectorial polarisation diagrams and complex polarisation analysis. Crampin and King [10] first time used the polarisation diagrams, PDs or hodograms to display the effect of upper mental anisotropy. In hodograms particle motion for successive time intervals is expressed on three component (3C) seismograms.

In rotated seismogram technique, Ando et al. [11] rotate the horizontal seismograms and select the polarizations that indicate different arrival time of shear waves. The classic vectorial polarisation diagrams demonstrate the presence of shear wave splitting. The initiatives of this technique Bernard and Zollo [12] plot the horizontal particle motion from origin that move linearly with time. The disadvantage of this method is neither the polarisation angles nor the time delays are easy to interpret. Vidale [13] introduced the complex polarisation technique in which the variation of eigen values and eigen vectors are interpreted in terms of variation in linear polarisation of shear wave particle motion, strike, angular and dip.

Automatic measuring technique consists of cross correlation techniques. This automatic analysis was initiated by Fukao [7]. He calculated the cross correlation of lag travel times i.e. shear wave splitting parameter and displays the results in polarisation diagrams. But the cross correlation method has some drawbacks like choosing a window for cross correlations and direct calculation of cross correlation for fast and slow shear wave.

Semi automatic method merges the advantage of both display technique and automatic technique. A shear wave

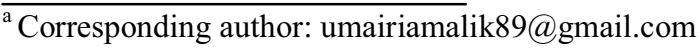

This is an Open Access article distributed under the terms of the Creative Commons Attribution License 4.0, which permits unrestricted use distribution, and reproduction in any medium, provided the original work is properly cited 
polarisation is estimated automatically and an expert system picks the arrival time which gives the initial measurement for visual adjustment [14].

Linearity techniques comprise aspect ratio method, linearity interval method, singular covariance matrix technique and singular value decomposition method (SVD). Sequentially rotating the seismograms for maximum linearity is called aspect ratio method by Shih et al. [15]. In aspect ratio method, possible fast and slow shear wave particle motion is projected onto orthogonal axes. This technique fails when the time delay between fast and slow shear wave is small. Aster et al.[16] selects the linearity interval by the magnitude of linearity vector in the direction of largest eigen value of variance matrix. Silver and Chan [17] propose the singular covariance matrix technique on the basis of complex polarisation technique of Vidale [13]. The non-zero eigen values are used for estimating the polarizations and time delays separately. This technique is robust for low frequency arrivals in the upper mantle however; it is less practical in the crust where the SWS is highly variable. In order to detect the hydrocarbon reservoirs from a borehole data [18] uses this technique for measuring the SWS automatically. An orthogonal matrix of three-component (3C) seismograms is decomposed into preferred polarisation with the aid of single value decomposition by [19]. The lag cross correlation method helps to find time delay between fast and slow shear wave. Although in the presence of noise this method gives appropriate results but hasn't been used widely.

Artificial neural network (ANN) techniques can also be used for the recognition of highly non- linear shear wave splitting behavior as observed by [20]. But ANN techniques don't provide sufficient evidence for shear wave splitting.

An industry standard, Radon transform (RT) has been attracted a lot of attention from last two decades. Radon transform, introduced by Johann Radon in 1917, is a mathematical basis for determining the structural properties of projected objects such as CT (computed tomography) scans and in magnetic resonance imaging (MRI) and variety of other radiological imaging techniques [21]. RT has been applied in a variety of fields like X-ray transmission computed tomography (TCT) or emission computed tomography (ECT), for prototype reconstruction problem, ultrasonic computed tomography (UCT), astronomy, reconstruction problem in electron microscopy, nuclear magnetic resonance (NMR), optics, micro crack detection and geophysics (seismology) [22].

Several applications of RT depict its importance in seismic data processing. RT can effectively remove random or correlated noise, enrich signal clarity and simultaneously constrain travel time and ray angles, while the inversion of RT is beneficial for phase isolation and spatial interpolation during the data reconstruction.

In seismic exploration, attenuations of unwanted events like surface waves and multiple compose a serious problem. The solution of this problem is move-out methods or curvature difference between unwanted events and the event of interest. But now RT is one of the solutions that helps not only to resolve these unwanted events by using the; linear (slant stack), parabolic (beam forming), hyperbolic (fan filtration) and generalized ( $\tau-p$ transform) but also for shear wave reflections from mantle interfaces, known as SS precursors phenomenon [23].

Near surface vertical shear wave profile is attained by using the Multichannel Analysis of Surface Waves (MASW). One of the important steps in MASW is to produce an image of dispersive image of energy in the frequency domain, so the dispersion curves can be obtained by picking peaks of dispersion energy. A high resolution linear radon transforms (LRT) is accomplished for Rayleigh waves energy, one of the surface waves. A differentiation of images of dispersion energy taken from real and synthetic data proves the preeminence of LRT as compare to slant stacking algorithm [24].

The ultimate target of seismic exploration is to generate the accurate images of subsurface to clearly investigate the hydrocarbon reservoirs. From the last 15 years Vertical Seismic Profile (VSP) surveys have been used for mapping fractures and fracture zones [25].

In VSP sources are placed on the surface while the receivers are placed vertically in the borehole at various known depths. VSP surveys are classified according to their geometry which is determined by source offset, borehole trajectory and receiver array depth. These geometeries find out dip range of interfaces and subsurface volume [26].

The elastic waves are propagated into lithosphere and anisotropy of mantle transition zone is found to split into two orthogonally polarized shear waves, $\mathrm{S}_{\mathrm{H}}$ and $\mathrm{S}_{\mathrm{V}}$. Shear wave splitting can give information about the salient points of anisotropy. However, the interaction of shear waves with subsurface structure is often complicated. Although fractures in hydrocarbon reservoirs are usually sub vertical. In case of zero-offset VSP most of the compressional energy comes in vertical component and horizontal components contain the shear and mode converted shear energy. Shear waves are recorded by using the receivers in borehole with zero-offset geometry and are not likely to travel vertically. So in this scenario measuring and interpreting the shear wave reflection is a non-trivial problem. A new approach Radon-Fourier transform (RFT) is proposed to measure the shear wave reflection by using the vertical component energy.

\section{Methodology}

The inhomogenity of earth proves that the elastic properties will change with depth. Seismic method employs the propagation of waves throughout the earth to locate the stratigraphy and structures. In exploration seismology, understanding of wave propagation is an important point and hence seismic modeling is an important tool [27]. Seismic modeling plays a crucial role in data acquisition, processing, interpretation and reservoir characterization. Synthetic seismograms should be generated in a process, called Forward Modeling for the validation of interpreted earth model out of seismic data. The seismic wave equations contain spatial and 
temporal derivatives are used to describe the seismic wave propagation in the subsurface. Different numerical methods are available to solve these wave equations. Some of the methods are: finite difference method, finite element method, boundary integral, pseudo-spectral, pseudo-element method, as well as Gaussian beam and Kirchhoff methods. Each method has its own assumptions, its appropriateness to problem and associated mathematical rigor [28]. Finite difference (FD) method is one of the most common methods and is theoretically based on the Taylor series expansion [27]. It discretizes the wave equation on a grid. Spatial derivatives are replaced by FD operator with neighboring points. The discretization of wave field is done in time domain, and wavefield for next step is calculated in general by using a Taylor series expansion.

\section{Mathematical Models and Solutions}

The 2D elastic wave equation in an isotropic medium [29] is

$$
\rho \frac{\partial^{2} u_{i}}{\partial t^{2}}=\frac{\partial \tau_{i j}}{\partial x_{j}}+F_{i}
$$

Where $u_{i}$ are particle displacements in two directions, $\left(u_{x}, u_{z}\right)$. For seismic waves they are typically known as radial and vertical components, respectively. Further $\tau_{i j}$ is stress tensor, $\rho$ is density, $t$ is time and $F_{i}$ is body force which is usually zero except for particular source. Therefore

$$
\begin{gathered}
\rho \frac{\partial^{2} u_{i}}{\partial t^{2}}=\frac{\partial \tau_{\mathrm{ij}}}{\partial \mathrm{x}_{\mathrm{j}}} \\
\text { Where } \tau_{i j}=C_{i j k l} \epsilon_{k l} \\
C_{i j k l}=\lambda \delta_{i j} \delta_{k l}+\mu\left(\delta_{i k} \delta_{j l}+\delta_{i l} \delta_{j k}\right) \\
\delta_{i j}=\left\{\begin{array}{l}
1 \text { for } i=j \\
0 \text { for } i \neq j
\end{array}\right\} \\
\epsilon_{i j}=\frac{1}{2}\left(\frac{\partial u_{i}}{\partial x_{j}}+\frac{\partial u_{j}}{\partial x_{i}}\right)
\end{gathered}
$$

The constitutive relation between stress and strain tensor is represented in equation (3) in which $\varepsilon_{k l}$ is a strain tensor and $C_{i j k l}$ is an elastic constant. Further consists of Lame parameters $\lambda, \mu$ and Knonecker delta function $\delta_{i j}$. Equation (6) represents the relation of displacement and strain. So the differential equations for 2D seismic wave propagation are as follows :

$$
\begin{aligned}
& \rho \frac{\partial u_{x}}{\partial t}=\frac{\partial \tau_{x x}}{\partial x}+\frac{\partial \tau_{x z}}{\partial z} \\
& \rho \frac{\partial u_{z}}{\partial t}=\frac{\partial \tau_{x z}}{\partial x}+\frac{\partial \tau_{z z}}{\partial z} \\
& \text { Where } \quad \tau_{x x}=(\lambda+2 \mu) \frac{\partial u_{x}}{\partial x}+\lambda \frac{\partial u_{z}}{\partial z} \\
& \tau_{z z}=(\lambda+2 \mu) \frac{\partial u_{z}}{\partial z}+\lambda \frac{\partial u_{x}}{\partial x} \\
& \tau_{x z}=\mu\left(\frac{\partial u_{x}}{\partial z}+\frac{\partial u_{z}}{\partial x}\right)
\end{aligned}
$$

Typically these equations are solved for particular velocities as $u=\frac{\partial u_{x}}{\partial t}, v=\frac{\partial u_{z}}{\partial t}$ and then the system is transformed into first order hyperbolic system as follows :

$$
\frac{\partial u}{\partial t}=c\left(\frac{\partial \tau_{x x}}{\partial x}+\frac{\partial \tau_{x z}}{\partial z}\right)
$$

$$
\begin{aligned}
& \frac{\partial v}{\partial t}=c\left(\frac{\partial \tau_{x z}}{\partial x}+\frac{\partial \tau_{z z}}{\partial z}\right) \\
& \frac{\partial \tau_{x x}}{\partial t}=(\lambda+2 \mu) \frac{\partial u}{\partial x}+\lambda \frac{\partial v}{\partial z} \\
& \frac{\partial \tau_{x z}}{\partial t}=\mu\left(\frac{\partial u}{\partial x}+\frac{\partial v}{\partial z}\right) \\
& \frac{\partial \tau_{z z}}{\partial t}=(\lambda+2 \mu) \frac{\partial v}{\partial z}+\lambda \frac{\partial u}{\partial x}
\end{aligned}
$$

As for many other approximations, finite difference method is a good method to approximate these differential equations. These differential equations are approximated by staggered grid discretization in space and time. 2D straggled grid finite difference wave propagation is as follows and $u$ is represented as $U, v$ as $V$, $\tau_{x x}$ as $R, \tau_{x z}$ as $S$ and $\tau_{z z}$ as $T$.

$$
\begin{gathered}
U_{i+\frac{1}{2}, j}^{k+\frac{1}{2}}=U_{i+1 / 2, j}^{k-1 / 2}+c_{i+\frac{1}{2}, j} \frac{\Delta t}{\Delta h}\left(R_{I+1 . j}^{k}-R_{i . j}^{k}\right)+ \\
c_{i+\frac{1}{2}, j} \frac{\Delta t}{\Delta h}\left(\mathrm{~S}_{i+\frac{1}{2}, j+\frac{1}{2}}^{k}-\mathrm{S}_{1+\frac{1}{2}, j-\frac{1}{2}}^{k}\right) \\
V_{i, j+1 / 2}^{k+1 / 2}=V_{i, j+1 / 2}^{k-1 / 2}+c_{i, j+\frac{1}{2}} \frac{\Delta t}{\Delta h}\left(\mathrm{~S}_{i+\frac{1}{2}, j+\frac{1}{2}}^{k}-\right. \\
\left.\mathrm{S}_{1-\frac{1}{2}, j+\frac{1}{2}}^{k}\right)+c_{i, j+\frac{1}{2}} \frac{\Delta t}{\Delta h}\left(\mathrm{~T}_{\mathrm{i}, \mathrm{j}+1}^{\mathrm{k}}-\mathrm{T}_{\mathrm{i}, \mathrm{j}}^{\mathrm{k}}\right) \\
R_{i, j}^{k+1}=R_{i, j}^{k-1}+(\lambda+2 \mu)_{i, j} \frac{\Delta t}{\Delta h}\left(U_{i+\frac{1}{2}, j}^{k+\frac{1}{2}}-\right. \\
\left.U_{i-\frac{1}{2}, j}^{k+\frac{1}{2}}\right)+\lambda_{i, j} \frac{\Delta t}{\Delta h}\left(V_{i, j+1 / 2}^{k+1 / 2}-V_{i, j-1 / 2}^{k+1 / 2}\right) \\
\mathrm{T}_{\mathrm{i}, \mathrm{j}}^{\mathrm{k}+1}=\mathrm{T}_{\mathrm{i}, \mathrm{j}}^{\mathrm{k}}+(\lambda+2 \mu)_{\mathrm{i}, \mathrm{j}} \frac{\Delta t}{\Delta h}\left(V_{i, j+\frac{1}{2}}^{k+\frac{1}{2}}-\right. \\
\left.V_{i, j-\frac{1}{2}}^{k+\frac{1}{2}}\right)+\lambda_{i, j} \frac{\Delta t}{\Delta h}\left(U_{i+\frac{1}{2}, j}^{k+\frac{1}{2}}-U_{i-\frac{1}{2}, j}^{k+\frac{1}{2}}\right) \\
\mathrm{S}_{i+\frac{1}{2}, j+\frac{1}{2}}^{k+1 / 2}=-\mathrm{S}_{1+\frac{1}{2}, j+1 / 2}^{k}+\mu_{i+\frac{1}{2}, j+\frac{1}{2}} \frac{\Delta t}{\Delta h}\left(V_{i+1, j+\frac{1}{2}}^{k+\frac{1}{2}}\right. \\
\left.V_{i, j+\frac{1}{2}}^{k+\frac{1}{2}}\right)+\mu_{i+\frac{1}{2}, j+\frac{1}{2}} \frac{\Delta t}{\Delta h}\left(U_{i+\frac{1}{2}, j}^{k+\frac{1}{2}}-U_{i+\frac{1}{2}, j}^{k+\frac{1}{2}}\right)
\end{gathered}
$$

In completion of one full time step $\Delta t$, update the velocities from stress or update the velocities from strain. The stability condition for homogenous medium depends on compressional velocity, shear wave velocity and variable Poisson's ration. Which are given as follows :

$$
\begin{aligned}
& V_{p} \frac{\Delta t}{\Delta h}<\frac{1}{\sqrt{2}} \\
& V_{p}=\sqrt{\frac{\lambda+2 \mu}{\rho}} \\
& V_{s}=\sqrt{\frac{\mu}{\rho}} \\
& v=\frac{\lambda}{2(\lambda+\mu)}
\end{aligned}
$$

The seismologists often treat a finite volume extracted from the Earth for a regional problem. Therefore, any artificial reflections from the given model boundaries should be minimized. For this purpose, an absorbing condition is required as a boundary to eliminate the reflected waves. Appropriate absorbing boundary conditions over a wide range of frequencies allow proper calculation not only for the wave propagation phase but also for the residual displacement field. If a boundary condition is not well implemented, the related reflected waves from the boundaries of the domain will affect the results strongly. Depending on the problem, different boundary conditions can be applied to the edges: free- 
surface conditions, absorbing boundaries [30] and Perfectly Matched Layer (PML) absorbing boundary [31] There are a lot of solutions to the boundary condition problems. The most popular boundary condition is "absorbing boundary condition" proposed by Engquist and Majda [32], and Clayton and Engquist [30].

Clayton-Engquist boundary conditions [30] are derived for numerical wave simulation that minimizes the artificial reflections from the isotropic gridded area. Thus the physical behavior of elastic wave propagation is efficiently described in an unbounded domain. These conditions are based on the paraxial approximations of elastic wave equations and also simple to apply for removing the boundary reflections over a wide range of incident angles. The Clayton Engquist boundary conditions are incorporated into 2D algorithms to remove the unwanted reflections from the sides and bottom of computational grid. These conditions absorb the horizontal propagating waves striking the side boundaries and vertically propagating plane waves striking the bottom boundary. The great advantage of these conditions is that they require only a few computations as compare to sponge boundary conditions, which require many points to be effective and full elastic waves have to be solved in the boundaries.

$$
\begin{aligned}
& U_{z}+\frac{U_{t}}{U_{s}}=0 \\
& V_{z}+\frac{V_{t}}{V_{p}}=0
\end{aligned}
$$

Where $\mathrm{U}$ and $\mathrm{V}$ are horizontal and vertical particle velocity; $V_{p}, V_{s}$ are $\mathrm{P}$ and $\mathrm{S}$ wave's velocities, respectively. Using the backward difference operator with respect to $\mathrm{z}$ and $\mathrm{t}$ in Madariaga-Virieux staggeredgrid scheme, equations (26) and (27) can be written as

$$
\begin{gathered}
\frac{U_{i, j}^{k-\frac{1}{2}}-U_{i, j-1}^{k-\frac{1}{2}}}{\Delta z}+\frac{U_{i, j}^{k+\frac{1}{2}}-U_{i, j-1}^{k-\frac{1}{2}}}{V_{s i, j} \Delta t}=0 \\
\frac{V_{i+\frac{1}{2}, j+1 / 2}^{k-1 / 2}-V_{i+\frac{1}{2}, j-1 / 2}^{k-1 / 2}}{\Delta z}+\frac{V_{i+\frac{1}{2}, j+1 / 2}^{k+1 / 2}-V_{i+\frac{1}{2}, j+1 / 2}^{k-1 / 2}}{V_{p_{i+\frac{1}{2}, j+1 / 2}}}=0
\end{gathered}
$$

Here $(i, j)$ is the space grid index; $k$ is the time grid index. From the formulae, particle velocities at time $k+1 / 2$ are calculated from the data at time $k-1 / 2$.

In seismology the generalized radon transform is defined as the integrals of amplitude

$$
u(\tau, q)=\int_{-\infty}^{+\infty} d(x, t) d x
$$

Where $\quad d(x, t)=\tau+q \varphi(x) \quad$ is the original seismogram and $u(\tau, q)$ will be its Radon transform; $x$ is a spatial variable like offset. $\varphi(x)$ defines the curvature upon which the transform curve is defined; $q$ is the slope of curvature; $\tau$ is the intercept time; and $t$ is two way travel time and $\omega$ is angular frequency [33]. For the relation between Fourier and Radon transforms, taking a Fourier transform of (30), as

$$
\begin{gathered}
f(q, \omega)=\int_{-\infty}^{+\infty} u(\tau, q) e^{i \omega \tau} d \tau \\
f(q, \omega)=\int_{-\infty}^{+\infty} d\left(x, t=\tau+q \varphi(x) e^{i \omega t} d \tau\right.
\end{gathered}
$$

\section{Results and Discussions}

The seismic reflection method for subsurface imaging has three stages: acquisition, processing and interpretation. Stage 1: A group of traces recorded from a common shot point, as shown in Fig.1, the geophones are distributed on the both sides of source also known as split spread or straddled spread. A single shot gather with 30 receivers are placed on the same surface.

Stage2: A single linear fracture model by specifying the horizontal distance $x$ on $x$ - axis as $2000 \mathrm{~m}$ and vertical depth $z$ in $y$ axis as $1000 \mathrm{~m}$. The layers have depth in increasing order. The first shot in $x$ direction is taken at $1000 \mathrm{~m}$ with the shot spacing of $25 \mathrm{~m}$. A frequency of 30 $\mathrm{Hz}$ and amplitude of signals that vary with time $t$ is described by using the ricker zero-phase wavelet approximates the dynamite source [35]. The $\mathrm{P}$ wave velocity must be in $(1000 \mathrm{~m} / \mathrm{s}$ to $5000 \mathrm{~m} / \mathrm{s}), \mathrm{S}$ wave velocity $(500 \mathrm{~m} / \mathrm{s}$ to $3500 \mathrm{~m} / \mathrm{s})$ and relative density in 1.0 to 2.7. A stable criterion for checking the $\mathrm{P}$ and $\mathrm{S}$ velocities are in range is Poisson's ration. The Poisson's ration must be less than 0.707 and assuming that the signal to noise ratio (SNR) is 1000 i.e. if the SNR is less than 1000 a noise is considered in model. Setting all these parameters according to specifications, the first order partial differential equations are approximated by staggered grid finite difference method. In finite difference, the various parameters of earth material like $\mathrm{Vp}=2000 \mathrm{~m} / \mathrm{s}, \mathrm{Vs}=700 \mathrm{~m} / \mathrm{s} \rho=2.5 \mathrm{Kg} / \mathrm{m}^{3}$ are defined on each node. The sampling rate $\Delta t$ is 2 millisecond and $\Delta x, \Delta z$ is $2.5 \mathrm{~m}$ that satisfy the stability condition i.e.

$$
\Delta t<\sqrt{2} \frac{\Delta x}{V_{\max }}
$$

The value of $\Delta x$ or $\Delta z$ depends on error criterion how closely spatial derivatives are need to be approximated by numerical method and limitation on $\Delta t$ is determined by courant number for stable2D finite difference numerical modeling. Such limitations on $\Delta x$ and $\Delta t$ make sure the stability of finite difference time stepping technique.

These partial differential equations are solved by setting the certain absorbing boundary conditions i. e. Clayton-Engquist (CE) boundary conditions. These conditions ensure the minimization of edge reflections of modeled seismograms. However, these conditions don't make the gridded area perfectly transparent as small reflections can be seen on common-source gather as shown in Fig.2. The Clayton Engquist boundary conditions assist to solve the partial derivative equations on the edges of model. The top layer is considered as free surface while the left, right and bottom boundaries are fixed for Clayton -Engquist boundary conditions.

After performing this forward modeling the waves are propagated into subsurface. The waves hit the subsurface and recorded by the receivers placed on surface. In Fig.2 the vertical axis is two way travel time while the horizontal axis is offset. The weak subsurface reflections are enhanced by a preprocessed step i.e. automatic gain control (AGC). The zero-offset VSP radial component shows $\mathrm{P}$ and $\mathrm{S}$ waves and the boundary reflections. 


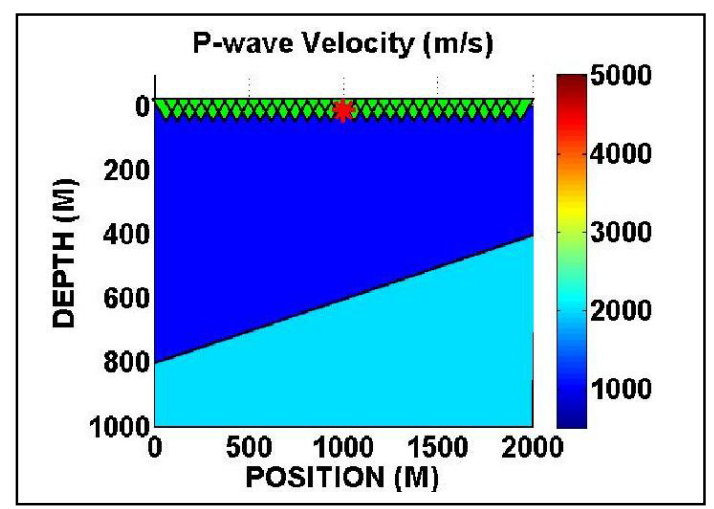

Figure 1. Velocity Model

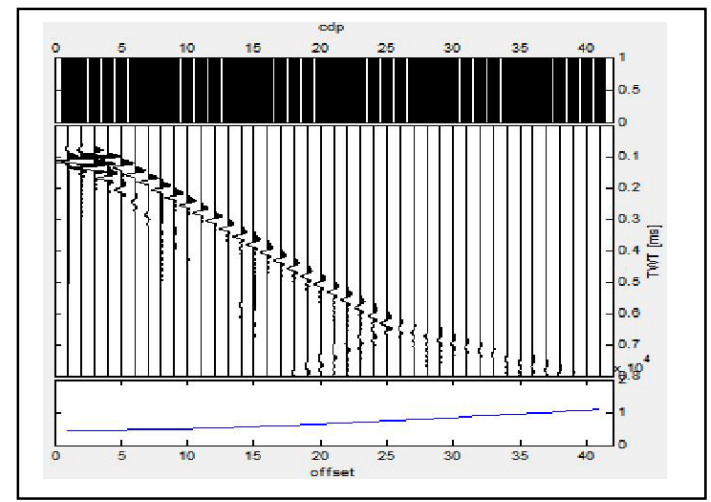

Figure 2. Radial Component

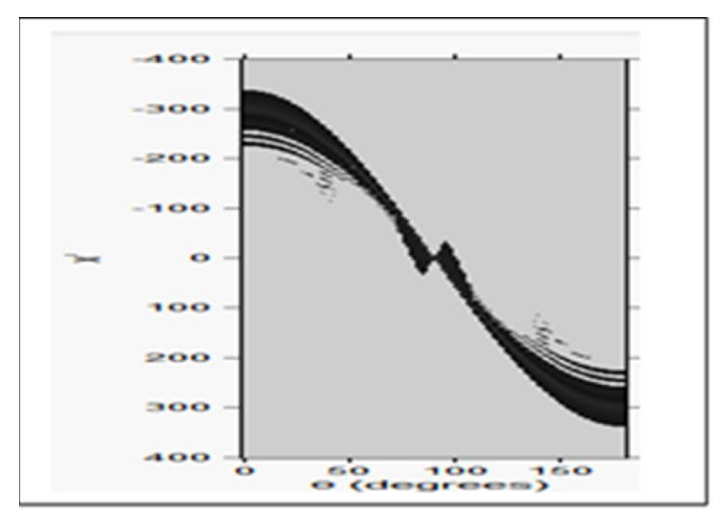

Figure 3. RFT Synthetic Data

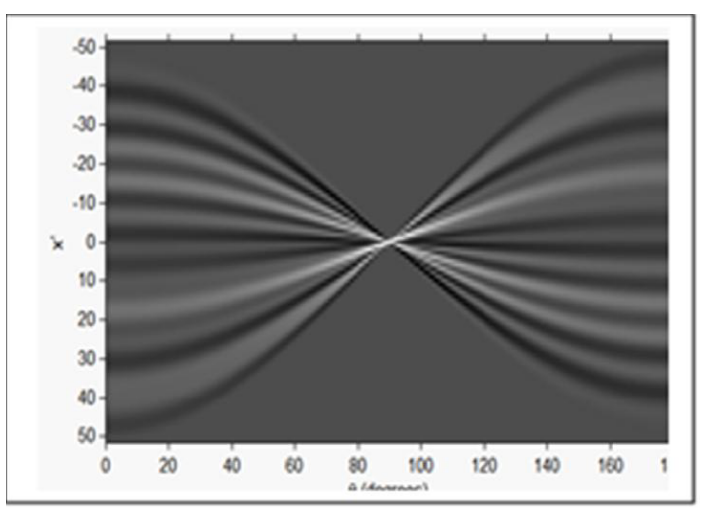

Figure 4. BF-Data
The Radon transform projects a Cartesian coordinate's reflection (offset, travel time) into radial coordinates. In Fig. 3 radon transform is applied in the offset-travel time data. The $x$-axis shows the values of angles $\Theta$ from 0 to 180 degree while the $y$-axis represents the perpendicular distance from line of reflection to origin. The midpoint is the distance from center and the angle at which the sum of intensities of wave peaks is taken. Later Fourier transform is employed on radon domain to observe the frequency spectrum and then synthesize back by multiplying with hyperbolic function makes the Radon-Fourier transform for the shear waves reflection. In Fig.4 the Radon-Fourier transform is applied on BF-data by taking its first 100 traces. The $x$ axis is the angle (degrees), and in $y$-axis the values in Radon domain, is a slope of line along with position.

The comparison of real and synthetic data on each values of $y$-axis is carried by using the root mean square (RMS) error of polynomial regression of first order. The minimum the RMS error of real and synthetic data would indicate the nearer shear wave reflection in RFT data. The first order polynomial regression equation is as follows:

$$
y=\alpha+\beta x+\varepsilon
$$

In equation (34), $y$ is dependent variable that are amplitude values, $\alpha$ is intercept i.e. the value of amplitude when all values of independent variable $x$ (angles) are set to zero. $\beta$ is regression coefficients for each independent variable and $\varepsilon$ is random error that follows the normal distribution with mean zero and unit standard deviation. The estimated regression equation for synthetic data for the corresponding $y$-axis values that have minimum RMS error is.

$$
\hat{y}=-2.239 e^{88}+7.839 e^{36} x
$$

With $\mathrm{SSE}=2.843 \mathrm{e}^{81}, \mathrm{R}^{2}=0.01057$, adjusted $\mathrm{R}^{2}=0.0538$ and $\mathrm{RMSE}=3.986 \mathrm{e}^{39}$. The term $-2.239 e^{88}$ indicates that at zero degree the amplitude is negative and with the increment in one degree amplitude increases $7.839 e^{36}$, Sum of Squares of Error (SSE) depicts the total variability in data and $0 \leq R^{2} \leq 1$. $R^{2}$ shows how much variability has been explained by the fitted model, the nearer to 1 the better the fitted model, however in the presence of noise it can be not to near to 1 or below the zero indicates that model is not fitted well to the data. RMS error is the fitted standard error. Now the equation for real data is as follows :

$$
y=-2.207 e^{37}+2.7983 e^{35} x
$$

With $\mathrm{SSE}=2.45 \mathrm{e}^{35}, \mathrm{R}^{2}=0.1363$, adjusted $\mathrm{R}^{2}=0.1315$ and $\mathrm{RMSE}=3.7 \mathrm{e}^{37}$.

On the basis of above results, at $10^{\text {th }}$ value in radon domain for all the theta values ( 0 to 180 degrees) the amplitude values are quite nearer. The RMS error of synthetic and real data shows that the difference between RMS error is not far apart. So in the presence of fracture the shear wave reflection in Radon domain for real and synthetic data is similar. 


\section{Conclusion}

Common depth point (CDP) traces are generated by using the forward modeling. Synthetic data have specific P wave velocity, $\mathrm{S}$ wave velocity and density. The weak subsurface reflections are enhanced by using the automatic gain control (AGC), a pre-processing method. The elastic waves are solved by using the finite difference method and with absorbing boundary conditions i.e Clayton Engquist (CE) boundary conditions. As the shear waves split in anisotropic medium, the reflectivity is observed into Radon-Fourier domain. Root mean square (RMS) error values in regression analysis are compared in synthetic and real data. The amplitude values from 0 to 180 degrees are compared for each trace. At the $10^{\text {th }}$ trace the Root Mean Square (RMS) error in synthetic and real BF-data are not far apart. This concludes that in presence of fracture at the $10^{\text {th }}$ trace the reflectivity is similar in synthetic and real data.

\section{References}

1. S. Crampin and S. Peacock, "A review of shearwave splitting in the compliant crack-critical anisotropic Earth," Wave Motion, 41, 59-77, (2005).

2. R. Bale, B. Gratacos, B. Mattocks, S. Roche, K. Poplavskii, and X. Li, "Shear wave splitting applications for fracture analysis and improved imaging: Some onshore examples," first break, 27, (2009).

3. S. Crampin and S. Peacock, "A review of the current understanding of seismic shear-wave splitting in the Earth's crust and common fallacies in interpretation," Wave motion, 45, 675-722, (2008).

4. C. M. Keith and S. Crampin, "Seismic body waves in anisotropic media: reflection and refraction at a plane interface," Geophys. J. Int, 49, 181-208, (1977).

5. M. Ando, Y. Ishikawa, and F. Yamazaki, "Shear wave polarization anisotropy in the upper mantle beneath Honshu, Japan," J. Geophys. Res: Solid Earth (1978-2012), 88, 5850-5864, (1983).

6. L. Vinnik, J. P. Montagner, N. Girardin, I. Dricker, and J. Saul, "Comment on "Shear-wave splitting to test mantle deformation models around Hawaii” by Kristoffer T. Walker, Götz HR Bokelmann, and Simon L. Klemperer," Geophys. Res. Lett, 30, (2003).

7. Y. Fukao, "Evidence from core-reflected shear waves for anisotropy in the Earth's mantle," (1984).

8. P. G. Silver and W. W. Chan, "Implications for continental structure and evolution from seismic anisotropy," (1988).
9. S. Crampin and Y. Gao, "A review of techniques for measuring shear-wave splitting above small earthquakes," Phys. Earth. Planet. In, 159, 1-14, (2006).

10. S. Crampin and D. W. King, "Evidence for anisotropy in the upper mantle beneath Eurasia from the polarization of higher mode seismic surface waves," Geophys. J. Int, 49, 59-85, (1977).

11. M. Ando, Y. Ishikawa, and H. Wada, "S-wave anisotropy in the upper mantle under a volcanic area in Japan," (1980).

12. P. Bernard and A. Zollo, "Inversion of nearsource S polarization for parameters of doublecouple point sources," B. Seismol. Soc. Am, 79, 1779-1809, (1989).

13. J. E. Vidale, "Complex polarization analysis of particle motion," B. Seismol. Soc. Am, 76, 1393-1405, (1986).

14. Y. Gao, P. Hao, and S. Crampin, "SWAS: A shear-wave analysis system for semi-automatic measurement of shear-wave splitting above small earthquakes," Phys. Earth. Planet. In, 159, 71-89, ( 2006).

15. X. R. Shih, R. P. Meyer, and J. F. Schneider, "An automated, analytical method to determine shear-wave splitting," Tectonophysics, 165, 271278, (1989).

16. R. C. Aster, P. M. Shearer, and J. Berger, "Quantitative measurements of shear wave polarizations at the Anza seismic network, southern California: Implications for shear wave splitting and earthquake prediction," J. Geophys. Res: Solid Earth (1978-2012), 95, 1244912473, (1990).

17. P. G. Silver and W. W. Chan, "Shear wave splitting and subcontinental mantle deformation," J. Geophys. Res: Solid Earth (1978-2012), 96, 16429-16454, (1991).

18. N. Teanby, J.-M. Kendall, and M. Van der Baan, "Automation of shear-wave splitting measurements using cluster analysis," B. Seismol. Soc. Am, 94, 453-463, (2004).

19. C.-F. Shieh, "Estimation of shear-wave splitting time using orthogonal transformation," Geophysics, 62, 657-661, (1997).

20. H. Dai and C. MacBeth, "Split shear-wave analysis using an artificial neural network," First Break, vol. 12, 605-613, 1994.

21. F. Colonna, G. Easley, K. Guo, and D. Labate, "Radon transform inversion using the shearlet representation," Acha, 29, 232-250, Sep (2010).

22. S. R. Deans, The Radon transform and some of its applications: Courier Corporation,(2007).

23. Y. J. Gu and M. Sacchi, "Radon transform methods and their applications in mapping mantle reflectivity structure," Surv. Geophys, 30, 327-354, (2009).

24. Y. Luo, J. Xia, R. D. Miller, Y. Xu, J. Liu, and Q. Liu, "Rayleigh-wave dispersive energy imaging using a high-resolution linear Radon 
transform," Pure. Appl. Geophys, 165, 903-922, May (2008).

25. C. Cosma, Vertical and Horizontal Seismic Profiling Investigations at Olkiluoto, 2001: Posiva, (2003).

26. J. Blackburn, J. Daniels, S. Dingwall, G. Hampden-Smith, S. Leaney, J. Le Calvez, et al., "Borehole Seismic Surveys: Beyond the Vertical Profile," Journal Oil Field Review, Schlumberger, 15, 15, (2007).

27. M. Nejati and H. Hashemi, "Migrated Exploding Reflectors in Evaluation of Finite Difference Solution for Inhomogeneous Seismic Models," (2012).

28. C. Sayers and S. Chopra, "Introduction to this special section: Seismic modeling," The Leading Edge, 28, 528-529, (2009).

29. Z. Yao and G. F. Margrave, "Elastic wavefield modelling in 3D by fourth-order staggered-grid finite difference technique."

30. R. Clayton and B. Engquist, "Absorbing boundary conditions for acoustic and elastic wave equations," B. Seismol. Soc. Am, 67, 1529-1540, (1977).

31. F. Collino and C. Tsogka, "Application of the perfectly matched absorbing layer model to the linear elastodynamic problem in anisotropic heterogeneous media," Geophysics, vol. 66, pp. 294-307, January 1, 20012001.

32. Engquist, B. and A. Majda (1979). Absorbing boundary conditions for the numerical solution of waves, Math. Comp. (in press).

33. Z. Cao, "Analysis and application of the Radon transform," in Masters Abstracts International, (2007).

34. R. H. Stolt and A. B. Weglein., Seismic Imaging and Inversion: Cambridge University Press, (2012).

35. A. Gholamy and V. Kreinovich, "Why Ricker wavelets are successful in processing seismic data: Towards a theoretical explanation," in CIES, 2014, IEEE Symposium on, 11-16 (2014) 